\title{
La presencia de las voces gitanas en los medios de comunicación españoles ${ }^{1}$
}

\section{The Romani voices' presence in Spanish media}

Gabriela Marques Gonçalves ${ }^{2}$

Resumen: Este trabajo objetiva debatir cómo la ausencia de las voces de determinados grupos sociales en el espacio público pueden perjudicar la efectividad de una democracia social plena, pensando la participación de las minorías en la esfera pública desde la comunicación. El trabajo de campo se basa en 20 entrevistas semi-estructuradas realizadas entre 2017 y 2019 en Barcelona (Cataluña) con la población gitana, la minoría étnica con más presencia en Europa. Abordaremos los productos mediáticos producidos sobre este colectivo, su participación en los media y los retos que ellos plantean en este área.

Palabras Clave: democracia; población gitana; espacio público; medios de comunicación; representación.

Abstract: This work aims to debate how the absence of some social groups' voices in the public space can prejudice the effectiveness of a broad social democracy. This work thinks about the minorities' participation in the public sphere since the communication field. The fieldwork is based on 20 semi-structured interviews realized between 2017 and 2019 in Barcelona (Catalonia) with the Romani population, the most present ethnical minority in Europe. We will discuss the media contents produced about this group, their participation in media and the challenges raised by them in this area.

Keywords: democracy; romani population; public space; media; representation.

l Este trabajo forma parte de una investigación realizada gracias a la beca de investigación doctoral de CAPES-MEC (Brasil).

2 Universitat Autònoma de Barcelona (UAB). Barcelona, Espanha.

https://orcid.org/0000-0002-9964-7757 E-mail: gabimarques@pm.me 


\section{Introducción}

Las teorías del espacio público están íntimamente relacionadas a las diferentes visiones sobre la democracia y su consolidación. Con el desarrollo de los medios de comunicación de masas, este espacio se ha vuelto más complejo, ganando diferentes modos de mediación y haciendo de los media actores importantes en la realización de los debates democráticos. Sin embargo, los sujetos sociales, cuyos discursos acceden a este espacio, son, comúnmente, los que detentan un saber validado por aquellos que tienen poder (CHAUÍ, 1986). Hay así una reducción del espacio democrático para algunas voces legitimadas y legitimadoras de lo que se entiende ser la vida en sociedad. Cuanto más reducido es el acceso a la producción de discursos en esta arena democrática, menos espacio hay para la diferencia. Desde las discusiones teóricas sobre la democracia deliberativa, Silveirinha (2005) trae al debate la propuesta de Young de pensar la democracia comunicativa.

Así, en esta presentación proponemos debatir cómo los sujetos autorizados son legitimados en el modelo de la democracia deliberativa, favoreciéndose aún más del contexto de los medios de comunicación de masas en lo que dice respeto a la formación de la opinión pública. Esta competencia del saber favorece a los grupos dominantes como las élites económicas y la élite política, dificultando el acceso a los grupos minoritarios a este espacio. Además de esto, el desarrollo de las tecnologías digitales no se muestra como solución para la diversificación de voces en estos espacios de deliberación. Como destaca Sodré (2002, p. 12-13), las nuevas tecnologías de la información conservan las "viejas estructuras de poder", aunque pueda al mismo tiempo acelerar lo que se entiende por democratización en los parámetros neoliberales. Todo ello nos hace preguntar cómo es posible garantizar la efectiva pluralidad de narrativas en el espacio público.

Centrando el debate en el contexto español, traeremos a la reflexión la realidad de la población gitana ${ }^{3}$ acerca del espacio que dispone en la

3 El contexto socio-histórico de la población gitana de España puede ser encontrado en otros trabajos del/de la autor/a. 
creación de las narrativas en los medios de comunicación. Este colectivo es la minoría étnica más numerosa de Europa y llegó al que hoy se reconoce como el territorio español a inicios del siglo XV. Desde su llegada, la población gitana ha sufrido persecuciones racistas sistematizadas por decretos reales y leyes que culminaron ya en el período democrático en la marginación socio-económica de gran parte del grupo, además del ocultamiento de su historia y de su cultura.

Intentando contribuir a los debates sobre esta invisibilización y buscando sacar a la luz los discursos de la población gitana, para el análisis aquí propuesto, recogeremos sus opiniones sobre cómo debe ser la presencia de este colectivo en los media españoles. Las experiencias nos muestran la dificultad en enumerar contenidos producidos por gitanos en los medios de comunicación y los desafíos del movimiento asociativo gitano en lo que se refiere a este campo. La muestra está compuesta por 20 sujetos mayores de 18 años residentes en la provincia de Barcelona, en Cataluña. La mitad trabaja en asociaciones o entidades gitanas y su nivel de escolarización va desde la primaria hasta el post grado. Son 10 hombres y 10 mujeres quienes componen el grupo de entrevistados cuyos discursos son analizados para este artículo.

\section{Democracia y espacio público}

Silveirinha (2005) describe la democracia como "una forma de lidiar con los desacuerdos entre ciudadanos que comparten una sola sociedad, pero no una sola cultura" (p. 4l). El abandono del macrosujeto histórico hizo que las minorías conquistaran un nuevo espacio en la democracia al reivindicar políticas relacionadas a la diferencia y a su representación. Así, nación y clase pasan a dividir lugar con cuestiones relacionadas con género, raza, etnicidad y sexualidad. Para la autora, ello constituyó el surgimiento de nuevos retos para los modelos democráticos vigentes, no sólo en relación al fin de la marginación de los grupos minoritarios, sino también a la promoción de la inclusión de las diferencias.

Silveirinha (2005) apunta tres tipos de democracia: la representativa liberal, la radical y la deliberativa, siendo esta última, según la autora, la 
más abierta a las cuestiones de los grupos minoritarios ya que la deliberación "es un proceso argumentativo en torno a las cuestiones relevantes y de reflexión sobre las alternativas disponibles” (p. 42-43). Según la autora, el diálogo posibilita la reflexión colectiva para llegar a un consenso sobre las reivindicaciones en cuestión. En la democracia deliberativa, la participación del ciudadano no se restringe a las elecciones, sino que se extiende al debate público en el intercambio de argumentos sobre diferentes cuestiones comunes a la vida en sociedad. Así, desde esta perspectiva, es necesario que haya un mayor alcance en relación a los actores que pueden efectivamente participar de la actividad política, es decir en las deliberaciones colectivas. En este proceso, la comunicación juega un rol importante ya que puede facilitar o impedir la toma de decisión.

Acabar con la marginación y promover la inclusión de las diferencias significa posibilitar "el acceso a las formas de participación en la vida colectiva” (SILVEIRINHA, 2005, p. 41-42) de forma horizontal a los diferentes grupos sociales, es decir, la participación dialógica activa en las discusiones públicas destinadas a deliberar decisiones. Esta perspectiva de democracia se apoya, entre otras, en las teorías del espacio público y de la comunicación de Habermas. Para este autor, desde la lectura de Silveirinha (2005), el espacio público incluye no sólo "los procesos políticos formalmente institucionalizados de opinión política y formación de la voluntad" (p. 49) sino también la esfera pública informal. Ello debe garantizar la equidad comunicativa para que haya de hecho una argumentación púbica dialógica entre los diferentes sujetos políticos.

\section{La equidad comunicativa}

La equidad comunicativa se posiciona como un elemento importante de la democracia deliberativa porque el foco no solo lo centra en el proceso de decisión sino que también incluye el proceso argumentativo que va a resultar en la decisión (SILVEIRINHA, 2005). Por ello, es necesario garantizar la participación igualitaria de los diferentes sujetos en los espacios de argumentación, lo que es imposibilitado constantemente por 
la concentración de poder (jurídico, político, económico y mediático), cuyas relaciones distorsionan la deliberación, lo que empuja a las minorías hacia los márgenes del debate público.

Esta marginación se debe también, según Silveirinha (2005), por un lado, a la resistencia de determinados sujetos miembros de grupos minoritarios a participar de los debates y, por otro, al desprecio hacia sus medios discursivos desde los grupos de poder, habiendo así una desigual capacidad en la participación en los espacios de argumentación. Ello da como resultado que el debate público siempre esté aliñado por la cultura dominante.

La resistencia a la participación y el desprecio a determinados discursos son resultado, por un lado, de lo que Chauí (2011, p. 19) llama el "discurso competente", reforzado por la idea de la "sociedad del conocimiento":

El discurso competente se confunde con el lenguaje institucionalmente permitido o autorizado, es decir, con un discurso en el que los interlocutores ya han sido previamente reconocidos como teniendo el derecho de hablar y oír, en el que los lugares y las circunstancias ya han sido predeterminadas para que sea permitido hablar y oír y, al fin, en el que el contenido y la forma ya han sido autorizados según los cánones de la esfera de su propia competencia (CHAUÍ, 2011, p. 19).

Este discurso competente es parte de una jerarquía organizacional que rige la vida en sociedad y que es reforzada constantemente por la figura del experto, aquél que ostenta el conocimiento sobre determinados asuntos. Según Chauí (2011), aquellos que no interiorizan las reglas de consolidación de este discurso pueden verse a si mismos como incompetentes y asociales. Así, la producción del saber no pertenece a todos, no es cosa pública, lo que imposibilita la participación igualitaria en los espacios de argumentación colectiva.

Para superar esta desigualdad, Silveirinha (2005) apunta la importancia de mirar la diferencia como recurso de comprensión del otro. Pero añadimos aquí, además, la necesidad de tener una consciencia histórica de los procesos de opresión y colonización que resultaron en esta 
desigualdad y, por lo tanto, en las necesidades específicas y particulares de determinados sujetos en el presente. Los privilegios históricos de determinados grupos sociales deben ser puestos sobre la mesa a la hora de la toma de decisiones. Ello porque hay "el refuerzo de la injusticia por la universalización de las normas de los grupos dominantes con base en políticas de distribución que son ciegas a la diferencia” (SILVEIRINHA, 2005, p. 54).

La grande crítica que la autora apunta sobre la democracia deliberativa basada en las ideas de Habermas es que se trata de "una concepción de democracia que privilegia una cultura de argumentación racional, una forma de discurso que no admite diferencia al habar y escuchar" (SILVEIRINHA, 2005, p. 55). Para superar esto, es necesario "incluir la representación y la participación directa de los grupos sociales en desventaja (...) (contrariando) el estatuto dominante y las jerarquías culturales" (SILVEIRINHA, 2005, p. 55-56). Como esta argumentación racional no está distribuída uniformemente entre todos los sujetos sociales, hace falta diversificar las formas de comunicación aceptadas en los espacios de deliberación.

Así, Young (1996 apud Silveirinha, 2005) propone la idea de democracia comunicativa en lugar de democracia deliberativa. En ella, la acción comunicativa resulta en la "reciprocidad asimétrica entre los sujetos” (YOUNG, 2000, apud SILVEIRINHA, 2005, p. 57), ya que se considera sus diferencias culturales y sus perspectivas sociales.

\section{Los medios de comunicación en la democracia}

El rol de los medios de comunicación de masas en la democracia es visto de diferentes maneras por los teóricos. Desde la perspectiva liberal, por ejemplo, la función de los medios es limitar el poder de los gobiernos y de las instituciones políticas. Para los autores de esta corriente,

(...) esta función es cumplida proporcionando al público información fiable considerada necesaria para formar una opinión informada. (...) las múltiples fuentes de información proporcionadas por los medios de 
comunicación son consideradas un suporte racional en la toma de decisiones (JAMAL, 2006, p. 31).

Ya los autores de la teoría crítica apuntan que la propiedad privada de los medios diseminaría la ideología de las clases dominantes. Para estos teóricos, "la propiedad privada de los medios de comunicación posibilita a los capitalistas controlar simultáneamente las instituciones políticas y mediáticas" (JAMAL, 2006, p. 30).

Según la perspectiva liberal, la concentración de los medios en las manos de algunos grupos empresariales sería equilibrada por la multiplicidad de flujos de comunicación y por las numerosas opciones proporcionadas por los media, lo que fortalecería la democracia (JAMAL, 2006). Los medios de comunicación de masas se presentan así como un espacio abierto a todos, sin embargo, "se funda en la hipótesis de que todo puede ser mostrado y dicho o de que todo es mostrable o decible, desde que establecidos criterios que autoricen quien puede mostrar y decir y quien puede ver y oír" (CHAUÍ, 1986, p. 31). El discurso competente es así reforzado por los medios de comunicación que nos convencen de "que estamos viendo y oyendo la producción colectiva de ideas o de valores" y que es necesario poseer los saberes vehiculados en ellos para poder "participar válidamente en la vida social" (CHAUí, 1986, p. 34-35).

A su vez, Silveirinha (2005), citando a Young, apunta que los medios de comunicación de masas son un reto para las teorías de la democracia, porque este espacio de argumentación pasa a ser mediado de forma compleja y no se da en una situación de interacción face a face. En este contexto,

la estructura del campo comunicativo, destinada a producir el sentimiento de la comunicabilidad plena, de la participación y de la comunidad, no es creada durante la práctica de la comunicación, no es un proceso de constitución recíproca de los interlocutores, sino antecede, regula, controla y predetermina la propia comunicación. El espacio es anterior a sus ocupantes, no es creado o recreado por ellos según la lógica peculiar del acto comunicativo (CHAUí, 1986, p. 31-32). 
$\mathrm{Al}$ no ser un proceso que se base en la práctica comunicativa de la reciprocidad de los interlocutores, se crea una homogeneidad en la perspectiva desde la que se tratan los temas de los contenidos emitidos. Tal homogeneidad no favorece la llamada democracia comunicativa ya que esta presupone "la diferencia, la discordancia y el conflicto" para que se pueda reconocer y abarcar las diferencias (SILVEIRINHA, 2005, p. 59). Otro factor que deriva en la falta de pluralidad es la concentración de medios de comunicación bajo el poder de algunos grupos financieros.

Ramonet (2012) cita el ejemplo de Francia, donde cinco grupos económicos dominan el sector de la prensa francesa de grande público. El autor también cita el dato de que un quinto de los miembros de los consejos de las principales empresas americanas están también en la dirección de los mayores medios de comunicación del país. Hay así, el "control capitalista sobre los medios de comunicación, considerados bienes como muchos otros, y en los que a menudo la transparencia de la estructura de propiedad está poco garantizada" (CAGÉ, 2016, p. 17).

Con el desarrollo tecnológico de la comunicación digital, el orden sociotecnológico se impuso sobre los procesos sociales cuyas "relaciones sociales de producción, por ejemplo, ya no consisten en una acción sobre las personas y las cosas, sino en una interacción entre las personas y la información" (ELHAJJI, 2005, p. 199). Esa tecnología hizo que las "instancias de mediación política, económica y social" se trasladaran de la dimensión espacial a la temporal (ELHAJJI, 2005, p. 193). En este nuevo contexto, la participación en los espacios de argumentación está basada en la instantaneidad e inmediatez en la elaboración de los discursos.

Las conexiones globales provocadas por las redes digitales y el carácter interactivo de estas tecnologías facilitaron el entrecruzamiento de los discursos de productores y receptores, que sumaron sus comentarios, fotos y videos a las narrativas producidas por aquellos que venían ostentando la competencia del discurso. Sin embargo, lo que podía aumentar el intercambio de argumentaciones, se transformó en un espacio más de los grupos dominantes. Ramonet (2012) califica como censura 
democrática este contexto de abundancia de información que nos impide acceder a otras informaciones, muchas de ellas relevantes para las luchas de las minorías.

Además de esto, es siempre importante destacar las brechas existentes en el uso de Internet y en su conectividad. Según el informe de la ONU sobre la medición de la sociedad de la información del 2017, el porcentaje de los hogares con conexión a Internet es dos veces mayor en los países desarrollados: "La probabilidad de que las personas accedan a Internet con regularidad en Europa es tres veces superior que en África, a lo que se suma la posibilidad de que los primeros gocen de mayores velocidades de acceso al conectarse" (ITU, 2017, p. 2).

Este contexto no favorable para el fortalecimiento de una democracia comunicativa puede ser contrarrestado por uno de los elementos comunicacionales apuntados por Young (2000 apud SILVEIRINHA, 2005) que ayuda a mantener la pluralidad en los espacios de argumentación que es la narrativa, el contar historias. Ello porque "la única solución para la conversación manipulativa o inapropiada es más conversación, para exponerla o corregirla, sea por un conjunto de razones, un modo de reconocimiento, una forma de argumentar o una narrativa” (YOUNG, 2000 apud SILVEIRINHA, 2005, p. 60). Por todo ello, la comunicación se coloca en un espacio central en la defensa del debate público como garante de una democracia efectivamente participativa y que respete las diferencias. Asegurar la participación a los grupos minoritarios en la producción de narrativas es así fundamental.

\section{Metodología}

Teniendo en cuenta el contexto presentado, nos proponemos analizar el caso específico de la población gitana de Cataluña que formó parte del grupo de entrevistados para este trabajo. El trabajo de campo fue desarrollado en la comarca de Barcelona entre los años 2016 y 2019, siendo la observación participante la principal herramienta metodológica para acercamiento a la muestra y para comprensión de la realidad analizada. 
A partir de este acercamiento, fue posible contactar y realizar las entrevistas para posterior análisis. En total, hemos contado con 20 personas, siendo 10 mujeres y 10 hombres, con edad entre 18 y 47 años y con diferentes niveles de educación formal. Entre las preguntas realizadas cuyas respuestas analizaremos en este trabajo están: ¿Hay algo sobre los gitanos en los medios de comunicación que sigues? ¿Qué le parece? ¿Sugeriría algo? ¿Algunos de los medios citados son producidos por gitanos?

Para las entrevistas semi-estructuradas se buscó crear un ambiente de diálogo distendido, facilitado en aquellos casos que la investigadora conocía previamente a la persona. Se intentó hacer entrevistas que tuvieran como cualidades "la flexibilidad de permitir al informante definir los términos de la respuesta y al entrevistador ajustar libremente las preguntas" (BARROS e DUARTE, 2008, p. 62). Este es un trabajo cualitativo por lo que el resultado estará relacionado "a la significación y a la capacidad que las fuentes tienen de dar informaciones fiables y relevantes sobre el tema de la investigación" (p.68) siempre relacionándolas con su mundo simbólico y social.

La sistematización de los datos se dio primeramente con la transcripción literal y completa de todas las entrevistas realizadas. Destacamos que hubo una preocupación por ser lo más fieles posible al discurso de las personas entrevistadas intentando reproducir las expresiones y modos de hablar de la muestra, con el objetivo de valorizar y visibilizar un legado lingüístico producido desde un contexto cultural propio. Las pequeñas modificaciones hechas resultaron simplemente de la adaptación del discurso hablado al escrito para una mejor comprensión del lector de este texto, ya que no se hizo una descripción de lenguajes corporales o demás herramientas propias de una conversación presencial.

La identificación de la muestra en el texto será hecha por una numeración que va de 1 a 20, totalizando el número de personas entrevistadas, seguida de $\mathrm{H}$ para hombres y $\mathrm{M}$ para mujeres y la edad correspondiente.

La participación en la producción de las narrativas mediáticas es importante para garantizar un espacio democrático y nuestro trabajo de 
campo se ha centrado en recoger las opiniones y actitudes del colectivo gitano. Ahora bien, es evidente que el análisis del modo en que los medios vienen mostrando a esta comunidad es un complemento indispensable para nuestro estudio. Para desarrollar esta cuestión emplearemos fuentes secundarias como se verá en el siguiente apartado.

\section{La población gitana y los medios de comunicación españoles}

Para comprender la realidad de la presencia de la población gitana en los medios de comunicación españoles hemos buscado diferentes estudios para análisis. El primer documento importante es el Informe sobre la diversidad y la igualdad en la televisión catalana en el año 2016, el último publicado por el Consejo de Audiovisual de Cataluña. Su objetivo es "analizar si los contenidos de las emisiones de televisión reflejan la diversidad de la sociedad actual y si el tratamiento es igualitario para los diferentes segmentos de la población” (2017, p. 13).

Los análisis fueron hechos desde dos categorías de contenidos: ficción y no-ficción. Sólo en la segunda se hicieron análisis referentes específicamente a la población gitana. Los resultados apuntaron que, en la TVC, las personas gitanas ocuparon 0,1\% del tiempo de palabra en cualquiera de los formatos. Se trata del menor porcentaje de presencia, si lo comparamos con el de blancos, árabes, asiáticos, personas con rasgos indígenas americanos, negros e indopakistanes. Por su parte, en TVE Catalunya el porcentaje es el mismo en los teleinformativos y presenta un ligero aumento hasta el $0,4 \%$ en las intervenciones en debates y entrevistas. Por último, en 8tv aparece idéntico índice, $0,1 \%$ en los teleinformativos, mientras que en los debates y entrevistas es inexistente.

Otro documento que nos ha servido es el informe anual ¿Periodistas contra el racismo? La prensa española ante el Pueblo Gitano, producido por la Unión Romaní desde 1995. Los datos que se presentan aquí son referentes al informe editado en 2018 que analiza 2.331 piezas publicadas el año anterior por 406 medios diferentes de la prensa escrita y 
digital. Del total del material, el 77,35\% de las piezas fueron valoradas como neutras, las negativas alcanzaron el $18,40 \%$ y las positivas descienden al 4,25\%, la cifra más baja desde que el informe empezó a ser publicado. Estas valoraciones positivas se concentran en los artículos de opinión, sumando la mitad de estos materiales.

En lo que se refiere a las fuentes gitanas, ellas estaban presentes en el 31,45\% de las piezas. Cataluña es la tercera región donde más se utilizaron estas fuentes, representando un 11,87\% del total de España. Cuando las comunidades autónomas son analizadas por separado, el $30,21 \%$ del material publicado en Cataluña presenta fuentes gitanas, un número ligeramente por debajo de la media nacional. Entre sus provincias, Barcelona es donde más textos se han publicado sobre la comunidad gitana $(93,74 \%)$. Si se analiza en relación a lo nacional, es la segunda provincia con más publicaciones (11,58\% del total), siendo la mayoría con valoración neutra $(80,74 \%)$.

El último documento que utilizamos es la tesis doctoral de Joan Oleaque (2014) sobre la representación de la población gitana en la prensa española. Fueron analizados tres diarios nacionales (ABC, El País y La Vanguardia) entre 1981 y 2010, en los cuales los gitanos aparecen como fuente en muy pocos casos. En ABC su voz aparece en dos ocasiones: una entrevista con el músico Manzanita en 1981 y en el reportaje sobre la muerte de Lola Flores en 1995 que recogió declaraciones dadas por la artista a lo largo de su vida.

El País es el medio que más evoca fuentes gitanas, sin embargo su uso no significa una imagen menos estereotipada de la población gitana. Así, en un reportaje del 1982, no aparece el nombre real ni completo de las fuentes gitanas, siendo utilizadas referencias que les ridiculizan como “'una madre (que) pregunta en tono patético' o como 'el patriarca Manolo” (OLEAQUE, 2014, p. 272). Además sus discursos se limitan a quejas y excusas. Ya en una noticia de 1995, "la voz de Los Otros llega a través de dos fuentes gitanas muy vulgarizadas" (OLEAQUE, 2014, p. 145), intensificando la relación con un origen callejero. Sólo en 2010 “se identificará más respetuosamente a las fuentes étnicas, pero no se 
recogen de ellas más que experiencias miserables, asistenciales y marginales como expulsados de Francia" (OLEAQUE, 2014, p. 272).

Finalmente, igual que en $\mathrm{ABC}$, la presencia de gitanos como fuente se da en solamente dos ocasiones en La Vanguardia, "con una visión mucho más oscura y marginal" ya que en ninguna de las dos ocasiones se da su versión sobre los hechos, sino que "intensifica una árida pintura suburbana sobre lo étnico y lo inmigrante” (OLEAQUE, 2014, p. 275). Ya en lo que se refiere a la autoría gitana, entre todos los textos analizados - considerados los más representativos del período abarcado -, solamente uno es escrito por un gitano, que es una crítica musical de 1989 publicada en ABC sobre un concierto de los músicos Paquera de Jerez y Camarón, en Madrid.

Esta poca presencia de gitanos en los medios de comunicación desde lugares que no estén marcados por el estereotipo se suma a lo que Ramonet (2012) llama "muro de información”, que hace que sea difícil encontrar determinados contenidos en un contexto de hiperabundancia de información.

En nuestro trabajo de campo, detectamos que muy pocos de los gitanos entrevistados acompañan con frecuencia el trabajo de sus pares que tienen una actuación más representativa o más constante en los medios de comunicación. En seis de las 20 entrevistas analizadas para este texto, la muestra no mencionó ninguna producción en medios de comunicación hecha por gitanos, aunque sí algunos de los entrevistados hicieron referencia a producciones artísticas en la música, las artes visuales y el cine. Entre los demás, la producción más mencionada fue el programa de radio semanal "Gitanos”, de Radio Nacional de España, conducido por Joaquín López Bustamante y Manuel Moraga, del cual cuatro entrevistados hicieron referencia. Sin embargo, sólo dos de ellos afirmaron acompañar el programa, y uno de los entrevistados destaca el día y horario de la emisión como un factor que dificulta el acceso.

... en Radio Nacional de España hay un programa, pero claro, el problema es que es domingo a las nueve de la mañana. O sea, ¿qué gitano o qué persona está despierta a un domingo a las nueve de la mañana, 
escuchando la radio, Radio Nacional de España? Claro es que es todo como más complicado, es como más obstáculos para que no llegues a ese programa que es muy bueno, muy bueno, y que habla especialmente de la cultura del pueblo gitano a nivel estatal, que lo llevan dos gitanos muy cultos y muy buenos, pero, claro, no llega a la mayoría de la gente, ni siquiera al pueblo gitano (E19H46).

Entre las otras producciones mencionadas, la mitad de nuestros entrevistados enunció publicaciones como revistas y periódicos, impresos o digitales, aunque algunos de ellos no han recordado sus títulos o sólo sabían de su existencia, sin seguir sus contenidos. Como ejemplo está el anuario ¿Periodistas contra el racismo ${ }^{4}$, mencionado por dos de los interlocutores. Además, una de las entrevistadas mencionó videos producidos por asociaciones como el documental Samudaripen (El holocausto olvidado $)^{5}$ sobre el holocausto gitano en Auschwitz, producido por Voces Gitanas y lanzado en 2016. Pero cabe destacar que todos estos entrevistados trabajan o colaboran en asociaciones gitanas, algunas a nivel nacional; otras, autonómico y otras, local.

... a nivel de publicación, hay que reconocer que la Fundación Secretariado Gitano tiene muy buenas publicaciones, pero se pierde la energía porque realmente no representa al pueblo gitano, realmente no. Es como muy gestionado a lo payo y eso no funciona, no funciona. Tiene que haber una buena gestión al mismo tiempo que la representación gitana esté garantizada porque si no no llega al público, ni en general ni gitano, solamente llega a la gente que está buscando información sobre gitanos para hacer estudios y estudios hay tantísimos que no valen para lo que se está pensando. (E19H46)

La evaluación de E19H46 sobre las publicaciones de Secretariado Gitano nos da una pista sobre por qué estos materiales no alcanzan efectivamente a la población gitana, lo que nos muestra el reto que el

4 Un resúmen de los informes está disponible en la página web de Unión Romaní (última visualización el 10/06/2019): [https://unionromani.org/periodistas-contra-el-racismo/].

5 El documental acompañó la visita de gitanos y gitanas de Cataluña al campo de concentración de Auschwitz, que forma parte del proyecto Dikh He Na Bister (Mira y no olvides). El documental está disponible en (última visualización en 04/04/2019): [https:/vimeo. $\mathrm{com} / 148087557]$. 
movimiento asociativo tiene en relación a sus estrategias de comunicación, de modo a producir contenidos que realmente interesen a la comunidad y también de encontrar modos de difusión que hagan que estos materiales alcancen a sus miembros.

Los otros medios a los cuales algunos entrevistados hacen referencia tienen relación con sus intereses personales, sea la religión, la dedicación a algún deporte, la música o las amistades. Así, radios evangélicas gestionadas por gitanos fueron mencionadas por dos entrevistados. Otro de ellos destacó reportajes televisivos sobre lucha greco-romana, a la que se dedicó profesionalmente por algunos años, y dos entrevistados más hicieron referencia a contenidos audiovisuales producidos por gitanos españoles o extranjeros.

La discusión sobre el tema de las producciones realizadas por gitanos y gitanas ha llevado a algunos entrevistados a mencionar experiencias propias en la producción de contenidos o, al menos, a mostrar interés en producir por si mismos contenidos relacionados con la población gitana. Se nota así, de algún modo, las posibilidades de intervención directa en los medios que parte de la muestra tiene con la producción de sus propios materiales mediáticos o culturales. Un ejemplo es el entrevistado que mencionó su deseo de cursar periodismo "para potenciar el mundo gitano en los medios de comunicación” (E6H25), aunque actualmente esté estudiando algo diferente, el grado en Trabajo Social. Otro ejemplo aparece ligado a la asociación Voces Gitanas, mencionada anteriormente. Creada en 2011, esta asociación trabaja especialmente en la creación de piezas radiofónicas y audiovisuales con jóvenes gitanas.

... hace poco estuvimos en la radio y propusimos hacer un programa de radio de gitanas, de chicas gitanas ... y sí que me gustaría, claro que me gustaría porque sería algo nuevo y algo que ... la gente que es gitana vería que podemos hacer más cosas. ... que se nos escuche un poco. No solamente diciendo somos gitanas, si no que se normalice un poco el tema. (E5M25)

La normalización de la presencia de la población gitana en los medios de comunicación es mencionada no sólo por E5M25. Esta demanda 
está relacionada a la producción de contenidos que no estén siempre relacionados a temas como discriminación y marginación pues lo que se plantea es que el protagonismo de los gitanos en la sociedad en diferentes ámbitos no quede oculto. Un ejemplo dado fue un programa cuya presentadora era Lolita Flores ${ }^{6}$.

... en la Televisión Española hay un programa de entrevistas super divertido, super natural, super ameno, super bien dirigido que la entrevistadora es una gitana, es Lolita Flores ... se ve muy dinámico y vi críticas del programa muy buenas, que hablaban de lo bien que lo hacía, la naturalidad que hacía las entrevistas ... la protagonista es Lolita Flores, como es algo bueno no la van a poner la etiqueta de gitana. Pues a estas cosas hay que poner la etiqueta de gitano, esto es gitano. Y es un ejemplo de televisión, pero como es tan bueno, pues no es un ejemplo de gitano porque lo gitano tiene que ser malo, tiene que ser casposo o tiene que ser flamenco, pero no puede ser algo normal ... Pues son ese tipo de cosas que debemos hacer, dejar que los gitanos participen con normalidad y no desde única y exclusivamente en el estereotipo gitano, o gustar de torcer ese estereotipo de ignorancia, de tontería, de atavismo, de cosas. Es buscar cosas diferentes. Esto creo que es un buen ejemplo de buena práctica (E16H36).

En general, se pudo notar en nuestra muestra una demanda para que se conozca mejor la historia y la cultura del Pueblo Gitano y una manera de hacerlo sería la producción de contenidos para los medios de comunicación o simplemente un cambio en la manera de producir el material sobre su pueblo. Por otro lado, dos de los entrevistados creen que esto no es lo ideal para acabar con la estigmatización de esta comunidad. Para uno de ellos, esto enmarcaría aún más las diferencias entre los gitanos y los no gitanos. En el segundo caso, la persona entrevistada entiende que lo más urgente es que se deje de enfocar en lo negativo. Opinión compartida por otros entrevistados que critican las generalizaciones que los medios de comunicación hacen sobre los gitanos, poniendo siempre los actos individuales en el marco de una práctica cultural y colectiva. Cuando se trata de los gitanos, las conductas de

6 Programa de la Televisión Española llamado "Lolita tiene un plan", de cuatro episodios, que se emitió en agosto de 2017 y se basaba en la realización de entrevistas. 
desviación son atribuidas por la sociedad mayoritaria al colectivo gitano (y no, al individuo). En muchos casos, se pide un cambio en el trabajo de los profesionales de comunicación.

Yo creo que si se dejara de publicar tantas cosas negativas no haría falta estar mostrando tanto la parte buena. Pero como la parte negativa sí que la hay, entonces por otra parte me gustaría que también enseñaran la otra, que eso no lo veo. ... la solución sería que las cosas negativas no le dieran tanto bombo porque también hay un montón de cosas negativas de otras culturas y no resaltan tanto. (E11M28)

Una iniciativa propuesta por uno de los entrevistados fue la realización de actividades de formación desde las entidades gitanas con profesionales de la comunicación. Sin embargo, en la opinión del entrevistado, los profesionales y empresas de comunicación están "muy cerrados a esa formación o a esa incorporación de este enfoque o perspectiva concreta, específica, puntual" (E19H46).

Finalmente, dos entrevistados comentaron que se deberían sancionar las malas prácticas en la representación del colectivo gitano y de otros colectivos en los medios de comunicación. Se subraya la importancia del código deontológico, pero también que se fiscalice si este código está siendo cumplido.

\section{Consideraciones finales}

Los relatos expuestos nos muestran como muchas veces la participación de voces gitanas en los medios de comunicación son vistos como discursos competentes solamente si se encajan en un formato que haya sido predeterminado anteriormente, como fue expuesto por Chauí (2011). Su participación es permitida siempre y cuando los lugares y las circunstancias de hablar y de oír ya hayan sido definidos con antelación. Aparte de esto, la presencia de las voces gitanas en los medios de comunicación es muy reducida, como nos muestran los estudios analizados, lo que dificulta la efectividad de participación en el espacio público. 
A pesar de estos datos sobre una baja participación en el debate público, hemos visto que algunas personas de la muestra ya han tenido en algún momento la oportunidad de participar en la producción de un contenido mediático. Aparte del entrevistado que es músico y utiliza regularmente sus canciones para hablar sobre su forma de vivir, otras dos entrevistadas también explican de qué les gustaría hablar en una producción mediática, especialmente enseñando desde su punto de vista qué es lo que hacen en su día a día. Hay un interés en enseñar su cultura y su modo de ver y vivir el mundo, yendo más allá de los temas que les reducen a los problemas que vive su comunidad.

Hemos visto, además, que no hay un seguimiento regular de contenidos producidos por la población gitana, aunque haya el interés por contenidos relacionados con su identidad como, por ejemplo, por acompañar noticias relacionadas con su colectivo.

Nuestros entrevistados tienen conciencia de los procesos de discriminación reforzados y/o creados por los mass media, al mismo tiempo que presentan un conocimiento sobre el funcionamiento de los medios suficiente para proponer formas de solucionar dichos problemas. Las frentes de actuación para garantizar la participación de la población gitana en este espacio de argumentación que forman parte los medios de comunicación se muestran muy diversas. Las experiencias recogidas aquí nos dan algunas herramientas para hacer el debate.

Finalmente, es importante destacar que, a partir de la actividad digital individual, sí se da el uso de herramientas como internet para hacer difusión de temas relativos a su identidad y a sus reivindicaciones. Resaltamos la importancia de proyectos y actividades realizados por diferentes asociaciones para fomentar el debate, la reflexión y la reivindicación de otras maneras de narrar qué es ser gitano.

\section{Referencias}

BARROS, Antônio. DUARTE, Jorge (Org.). Métodos e técnicas de pesquisa em comunicação. São Paulo: Atlas, 2008.

CAGÉ, Julia. Salvar los medios de comunicación. Barcelona: Editorial Anagrama, 2016. 
CHAUÍ, Marilena. Conformismo e resistência: Aspectos da cultura popular no Brasil. São Paulo, Brasil: Brasiliense, 1986.

CHAUÍ, Marilena. Cidadania cultural: o direito à cultura. São Paulo, Brasil: Editora Fundação Perseu Abramo, 2006.

CONSELL DE L'AUDIOVISUAL DE CATALUNYA. Informe sobre la diversidad y la igualdad en la televisión. Año 2016. Barcelona, España: CAC, 2017.

ELHAJJI, Mohammed. Comunicação, cultura e conflitos: uma abordagem conceitual. En: PAIVA, Raquel; BARBALHO, Alexandre (Org.). Comunicação e cultura das minorias (pp. 189-205). São Paulo, Brasil: Paulus, 2005.

INTERNATIONAL TELECOMMUNICATIONS UNION. Informe sobre la medición de la sociedad de la información de 2017 - Resumen. Recuperado el 08 de mayo de 2018 de https:/www.itu.int/en/ITU-D/Statistics/Documents/publications/misr2017/ MISR2017_ES_S.pdf

JAMAL, Amal. The culture of media consumption among national minorities: The case of Arab society in Israel. Nazareth, Israel: I'lam - Media Center for Arab Palestinians in Israel, 2006.

OLEAQUE, Joan Moreno. Los gitanos en la prensa española: Variación y reiteración de los planteamientos de los diarios ABC, El País, y La Vanguardia en la representación de los gitanos como grupo (1981-2010). Tesis Doctoral. Valencia, España: Universitat de València, 2014.

SILVEIRINHA, Maria João. Democracia e reconhecimento: repensar o espaço público. En: PAIVA, Raquel; BARBALHO, Alexandre (Org.). Comunicação e cultura das minorias (pp. 41-69). São Paulo, Brasil: Paulus, 2005.

SODRÉ, Muniz. Antropológica do Espelho: uma teoria da comunicação linear e em rede. Petrópolis, Brasil: Vozes, 2002.

\section{Sobre los autores:}

Gabriela Marques Gonçalves - Doutora em Comunicação Audiovisual e Publicidade pela Universidad Autónoma de Barcelona.

Data de submissão: 28/03/2020

Data de aceite: 15/06/2020 